\title{
Multicenter evaluation of a syndromic rapid multiplex PCR test for early adaptation of antimicrobial therapy in adult patients with pneumonia
}

Céline Monard', Jonathan Pehlivan², Gabriel Auger ${ }^{3,4}$, Sophie Alviset $^{5}$, Alexy Tran Dinh ${ }^{6,7}$, Paul Duquaire ${ }^{1}$, Nabil Gastli ${ }^{8}$, Camille d'Humières ${ }^{9,10}$, Adel Maamar ${ }^{11,12}$, André Boibieux ${ }^{13}$, Marion Baldeyrou ${ }^{14}$, Julien Loubinoux ${ }^{15}$, Olivier Dauwalder ${ }^{16,17}$, Vincent Cattoir ${ }^{3,18,19}$, Laurence Armand-Lefèvre ${ }^{9,10}$, Solen Kernéis ${ }^{5,10^{*}}$ and the ADAPT study group

\begin{abstract}
Background: Improving timeliness of pathogen identification is crucial to allow early adaptation of antibiotic therapy and improve prognosis in patients with pneumonia. We evaluated the relevance of a new syndromic rapid multiplex PCR test (rm-PCR) on respiratory samples to guide empirical antimicrobial therapy in adult patients with communityacquired pneumonia (CAP), hospital-acquired pneumonia (HAP), and ventilator-acquired pneumonia (VAP).
\end{abstract}

Methods: This retrospective multicenter study was conducted in four French university hospitals. Respiratory samples were obtained from patients with clinical and radiological signs of pneumonia and simultaneously tested using conventional microbiological methods and the rm-PCR. A committee composed of an intensivist, a microbiologist, and an infectious diseases specialist retrospectively assessed all medical files and agreed on the most appropriate antimicrobial therapy for each pneumonia episode, according to the results of rm-PCR and blinded to the culture results. The rm-PCR-guided antimicrobial regimen was compared to the empirical treatment routinely administered to the patient in standard care.

Results: We included 159 pneumonia episodes. Most patients were hospitalized in intensive care units $(n=129,81 \%)$, and episodes were HAP $(n=68,43 \%), \operatorname{CAP}(n=54,34 \%)$, and VAP $(n=37,23 \%)$. Conventional culture isolated $\geq 1$ microorganism(s) at significant level in 95 (60\%) patients. The syndromic rm-PCR detected at least one bacteria in 132 (83\%) episodes. Based on the results of the rm-PCR, the multidisciplinary committee proposed a modification of the empirical therapy in 123 (77\%) pneumonia episodes. The modification was a de-escalation in 63 (40\%), an escalation in 35 (22\%), and undetermined in 25 (16\%) patients. In microbiologically documented episodes $(n=95)$, the rm-PCR increased appropriateness of the empirical therapy to 83 (87\%), as compared to 73 (77\%) in routine care.

(Continued on next page)

\footnotetext{
* Correspondence: solen.kerneis@aphp.fr

${ }^{5}$ Equipe Mobile d'Infectiologie, APHP, Hôpital Cochin, Centre Université de

Paris, Paris, France

10IAME, INSERM, Université de Paris, Paris, France

Full list of author information is available at the end of the article
}

\section{$\triangle B M C$}

(c) The Author(s). 2020 Open Access This article is licensed under a Creative Commons Attribution 4.0 International License, which permits use, sharing, adaptation, distribution and reproduction in any medium or format, as long as you give appropriate credit to the original author(s) and the source, provide a link to the Creative Commons licence, and indicate if changes were made. The images or other third party material in this article are included in the article's Creative Commons licence, unless indicated otherwise in a credit line to the material. If material is not included in the article's Creative Commons licence and your intended use is not permitted by statutory regulation or exceeds the permitted use, you will need to obtain permission directly from the copyright holder. To view a copy of this licence, visit http://creativecommons.org/licenses/by/4.0/ The Creative Commons Public Domain Dedication waiver (http://creativecommons.org/publicdomain/zero/1.0/) applies to the data made available in this article, unless otherwise stated in a credit line to the data. 
(Continued from previous page)

Conclusions: Use of a syndromic rm-PCR test has the potential to reduce unnecessary antimicrobial exposure and increase the appropriateness of empirical antibiotic therapy in adult patients with pneumonia.

Keywords: Antimicrobials, Antimicrobial stewardship, Pneumonia, Multiplex PCR, Syndromic tests, Biofire ${ }^{\circledR}$ FilmArray ${ }^{\circledR}$

\section{Background}

Inadequate and delayed empirical treatments are strong predictors of mortality in sepsis [1, 2]. Therefore, in pneumonia patients, international guidelines state that an attempt should be made to obtain respiratory samples and recommend to start early empirical treatment while awaiting for the results of culture and antimicrobial susceptibility testing (AST) [3]. For severe patients or those with risk factors of multidrug-resistant organisms (MDRO), the empirical treatment should include a broad-spectrum antibiotic [4, 5]. However, conventional microbiological techniques have a low sensitivity, particularly on microbiological samples collected in non-intubated patients and in case of prior exposure to antibiotics [6,7]. The lack of a reliable microbiological diagnosis thus prevents from deescalating the empirical regimen in a large proportion of patients [8].

Identification of causative microorganisms provides the potential to target antibiotic therapy, but the turnaround time from microbiological sampling to AST usually requires at least $48 \mathrm{~h}$. New molecular diagnostic tools aim at shortening this time. Syndromic rapid multiplex PCR (rm-PCR) can be used for simultaneous detection of multiple organisms and resistance markers in a specific clinical context, within a few hours [9]. Alongside with antimicrobial stewardship (AMS), the use of rm-PCR has been shown to significantly decrease time-to-appropriate therapy and optimize clinical and economic outcomes $[10,11]$. During a previous evaluation in community-acquired pneumonia, a rm-PCR assay achieved pathogen detection in $87 \%$ of patients compared to $39 \%$ using culture-based methods [12]. In addition, in this population, molecular testing had the potential to lead to a de-escalation in the number and/or spectrum of initial empirical antibiotics in $77 \%$ of patients.

The BioFire ${ }^{\circledast}$ FilmArray ${ }^{ø}$ Pneumonia Panel (bioMerieux S.A., Marcy-l'Etoile, France) is a novel assay able to simultaneously identify 27 of the most common pathogens involved in lower respiratory tract infections (semi-quantitative results for 11 Gram-negative and 4 Gram-positive bacteria, qualitative results for 3 atypical bacteria and 9 viruses) as well as 7 antibiotic resistance genes (Fig. 1). Two studies have found excellent agreement between this molecular method and standard culture [13, 14].

Our main objective was to estimate the potential impact of this new syndromic rm-PCR assay on early adaptation of empirical antimicrobial therapy in adult patients with pneumonia.

\section{Methods}

\section{Settings and participants}

Between July and December 2018, 11 French university hospitals participated in a pre-commercialization evaluation of the investigative-use-only (IUO) version of the rm-PCR BioFire ${ }^{\bullet}$ FilmArray $^{\oplus}$ Pneumonia Panel (bioMerieux S.A., Marcy-l'Etoile, France). A total of 515 respiratory samples (sputa, endotracheal aspirations (ETA), blind bronchial sampling (BBS), and bronchoalveolar lavages (BAL)) were tested simultaneously, using conventional techniques and the rm-PCR. The syndromic rm-PCR demonstrated high agreement with conventional culture in this panel of patients $[14,15]$.

Four centers (Bichat-Paris, Cochin-Paris, Rennes, and Lyon) were further selected to participate in the present sub-study. Criteria for patient inclusion were as follows: (1) age $\geq 18$ years and (2) presence of clinical and radiological criteria for pneumonia according to the IDSA guidelines: new lung infiltrate on a chest X-ray and evidence that the infiltrate was of an infectious origin, i.e., at least two of three clinical features (fever greater than $38{ }^{\circ} \mathrm{C}$, leukocytosis or leukopenia, and purulent secretions) [16]. Criteria for pneumonia were evaluated by two clinical investigators in each center. Communityacquired pneumonia (CAP), hospital-acquired pneumonia (HAP), and ventilator-associated pneumonia (VAP) were included. HAP was defined as pneumonia occurring $48 \mathrm{~h}$ or more after admission, which was not incubating at the time of admission and not associated with mechanical ventilation [4]. VAP referred to pneumonia arising $>48 \mathrm{~h}$ after endotracheal intubation $[4,16]$. CAP included all episodes of pneumonia acquired outside of the hospital setting. Even if not strictly included in the most recent definition of CAP [17], we also included patients with immunocompromising conditions.

\section{Microbiology methods}

Respiratory specimens were routinely analyzed in each local microbiology laboratory according to current recommendations of the French Standard Guidelines in Medical Microbiology (REMIC) [18]. In brief, sputum and ETA samples were digested and diluted according to sample types. Then, they were streaked on 


\begin{tabular}{|c|c|}
\hline Bacteria & Viruses \\
\hline Acinetobacter calcoaceticus-baumannii complex & Adenovirus \\
\hline Serratia marcescens & Coronavirus \\
\hline Proteus spp. & Human Rhinovirus/Enterovirus \\
\hline Klebsiella pneumoniae group & Human Metapneumovirus \\
\hline Enterobacter aerogenes & Influenza A \\
\hline Enterobacter cloacae complex & Influenza B \\
\hline Escherichia coli & Parainfluenza Virus \\
\hline Haemophilus influenzae & Respiratory Syncytial Virus \\
\hline Moraxella catarrhalis & Middle East Respiratory Syndrome Coronavirus \\
\hline Pseudomonas aeruginosa & \\
\hline $\begin{array}{l}\text { Staphylococcus aureus } \\
\text { Streptococcus pneumoniae } \\
\text { Klebsiella oxytoca } \\
\text { Streptococcus pyogenes } \\
\text { Streptococcus agalactiae }\end{array}$ & \multirow{2}{*}{$\begin{array}{l}\text { Antibiotic Resistance Markers } \\
\text { bla }_{\mathrm{CTX}-\mathrm{M}}(\mathrm{ESBL}) \\
\text { bla }_{\mathrm{IMP}} \text { (Carbapenemase) } \\
\text { bla }_{\mathrm{KPC}} \text { (Carbapenemase) } \\
\text { mecA/C - MREJ (methicillin resistance) } \\
\text { bla }_{\mathrm{NDM}} \text { (Carbapenemase) } \\
\text { bla }_{\mathrm{OXA}-48-\mathrm{like}} \text { (Carbapenemase) } \\
\text { bla }_{\mathrm{VIM}} \text { (Carbapenemase) }\end{array}$} \\
\hline $\begin{array}{l}\text { Atypical Bacteria } \\
\text { Legionella pneumophila } \\
\text { Mycoplasma pneumoniae } \\
\text { Chlamydophila pneumoniae }\end{array}$ & \\
\hline
\end{tabular}

Fig. 1 Pathogens targeted by the BioFire ${ }^{\oplus}$ FilmArray ${ }^{\oplus}$ Pneumonia Panel

recommended plates according to semi-quantitative patterns and incubated for 2 days in $\mathrm{CO}_{2}$ and aerobic conditions. Results of standard culture were expressed in colony-forming unit $(\mathrm{CFU}) / \mathrm{mL}$. The thresholds for positivity on culture were $\geq 10^{3} \mathrm{CFU} / \mathrm{mL}$ for $\mathrm{BBS}, \geq 10^{4}$ $\mathrm{CFU} / \mathrm{mL}$ for $\mathrm{BAL}, \geq 10^{5} \mathrm{CFU} / \mathrm{mL}$ for ETA, and $\geq 10^{7}$ $\mathrm{CFU} / \mathrm{mL}$ for sputa [19]. Diagnostic tests for viruses and atypical bacteria were conducted only if requested by the physician in charge.

Respiratory samples were simultaneously tested with the IUO version of the BioFire ${ }^{\bullet}$ FilmArray $^{\bullet}$ Pneumonia Panel according to the manufacturer's instructions directly on native respiratory samples. The IUO version is identical to the final FDA-cleared and CE-marked version. The system integrates sample preparation, nucleic acid extraction and purification, amplification, detection, and analysis, with a total run time of about $1 \mathrm{~h}$. Results of the syndromic rm-PCR are expressed as semiquantitative results $\left(10^{4}\right.$ to $\left.\geq 10^{7}\right)$ in DNA-copies/mL for commonly culturable bacteria and as qualitative results (presence/absence) for resistance genes, viruses, and atypical bacteria. Bacteria found under the threshold of $10^{3.5}$ copies $/ \mathrm{mL}$ are not reported on the final $\mathrm{rm}$-PCR report.

\section{Data sources}

Clinical and demographical data were retrospectively obtained from the electronic medical records of each patient. Investigators collected demographic characteristics (age, gender) and medical data such as medical history, classification of pneumonia (VAP, HAP, or CAP), severity scores, and antibiotics prescribed.

In each study center, a multidisciplinary committee composed of an intensivist, an infectious diseases specialist, and a clinical microbiologist was formed. During a face-to-face meeting, the local multidisciplinary committee retrospectively reviewed medical files of all pneumonia patients, including patients' medical history, previous antimicrobial treatments received, previous microbial colonization and risk factors for MDRO carriage, clinical parameters (e.g., fever, hemodynamics, and respiratory parameters), results of standard biological analyses, and chest imaging. For each episode, the result of the syndromic rm-PCR was presented to the committee, blinded to (1) the direct examination of the sample, (2) the results of the standard culture and AST, and (3) the empirical therapy administered in routine care. Guided by the rm-PCR results, the multidisciplinary committee agreed on the most appropriate antimicrobial therapy for each pneumonia episode.

\section{Endpoints}

The antimicrobial therapy proposed by the multidisciplinary committee based on syndromic rm-PCR results (PCR-guided therapy) was compared to the empirical therapy actually delivered to the patient in routine care 
(empirical therapy). The primary endpoint was the number of pneumonia episodes in which PCR-guided therapy differed from empirical therapy. The secondary endpoints were as follows: (1) the number of de-escalations of the antibiotic regimen based on syndromic rm-PCR results and (2) the number of episodes in which PCRguided therapy would be active on pathogens isolated at significant threshold on culture. Both primary and secondary endpoints were evaluated by an independent central committee composed of an intensivist and an infectious disease specialist from two different university hospitals (SK and CM).

De-escalation was defined according to previous studies as discontinuation of any companion drug and/or switch of the pivotal drug to a narrower-spectrum antibiotic [20,21]. Ranking of pivotal drugs was determined according to their antibacterial spectrum and putative ecological impact and defined either within the same antibiotic family or from a family to another (e.g., vancomycin to oxacillin). $\beta$-lactams were categorized into six groups, as previously described by Weiss et al. [22] (Fig. 2). In several cases (e.g., switch from piperacillin/tazobactam to a fourth-generation cephalosporin), as hierarchy of antibiotics could not be established, the antibiotic change was qualified as "undetermined." If a companion drug was stopped but a new one was started, the antibiotic change was also considered as "undetermined."

Definitive results of standard culture and AST were considered by the central committee to state on the susceptibility of pathogens to the PCR-guided therapy. This secondary endpoint was evaluated only in microbiologically documented pneumonia episodes. Only pathogenic microorganisms cultured at significant levels on culture were considered as documentation. Coagulase-negative staphylococci (CoNS) and Enterococcus spp. were considered as non-pathogenic.

\section{Statistical methods and ethics}

All data were anonymously collected and stored on a secured database. Descriptive analysis was performed using the R software (3.3.2, R Foundation for Statistical Computing, Vienna, Austria). Due to relatively small numbers and the retrospective design of our study, we did not perform a formal statistical analysis, but rather present the description of data. For each variable, when data were missing, they were excluded from the calculation of percentages. According to the French legislation, this study was approved by the Ethics Committee of the French Society of Infectious Diseases (Société de Pathologie Infectieuse de Langue Française), n'2019-0902, and declared to the French national data protection commission (Commission Nationale de l'Informatique et des Libertés, CNIL), n¹9-213.

\section{Results \\ Patients}

Among 170 pneumonia episodes included in the precommercialization evaluation in the four investigating centers, 159 were retained for final analysis. Exclusion criteria were as follows: age $<18$ years $(n=2)$ and criteria for pneumonia not fulfilled $(n=9)$. A total of 129 patients $(81 \%)$ were hospitalized in intensive care units (ICUs) at inclusion, and their median [IQR] SOFA score was 6 [2.5-8.5]. Overall in-hospital mortality was $28 \%$

\begin{tabular}{|lll|}
\hline Rank & Molecule(s) & $\begin{array}{c}\text { Spectrum and resistance- } \\
\text { promoting potential }\end{array}$ \\
$\mathbf{1}$ & Amoxicillin & \\
$\mathbf{3}$ & Amoxicillin + clavulanate & Third-generation cephalosporin \\
$\mathbf{4}$ & Ureido/carboxy-penicillin & Piperacilin + Tazobactam \\
& Ticarcilin + clavulanate & \\
& Fourth-generation cephalosporin, \\
$\mathbf{5}$ & Antipseudomonal third-generation cephalosporin \\
$\mathbf{6}$ & Ertapenem & \\
& Imipenem & \\
\end{tabular}

Fig. 2 Consensual ranking of $\beta$-lactams according to both their spectrum and their resistance-promoting potential according to Weiss et al., Clinical Microbiology and Infection 2015 "Elaboration of a Consensual Definition of De-Escalation Allowing a Ranking of $\beta$-Lactams" 
(45/159). Pneumonia episodes were classified as HAP $(n=68,43 \%)$, CAP $(n=54,34 \%)$, and VAP $(n=37,23 \%)$. Microbiological samples were as follows: ETA $(n=71$, $45 \%)$, sputum samples $(n=33,21 \%)$, BAL $(n=34,21 \%)$, and BBS $(n=21,13 \%)$. Other demographic data are reported in Table 1.

\section{Microbiology and routine antimicrobial therapy}

Conventional culture isolated $\geq 1$ microorganism(s) at significant level in 95 episodes (60\%): 27/54 (50\%) in CAP, 40/68 (59\%) in HAP, and 28/37 (76\%) in VAP. The syndromic rm-PCR detected at least one bacteria in 132 (83\%) episodes; a viral co-infection was detected in 16 episodes and a virus without bacteria in 6 episodes. Among the 64 undocumented episodes on culture, the syndromic rm-PCR identified a microorganism in $21 \mathrm{ep}-$ isodes (bacteria in 19 episodes and viruses in 2 episodes). The main pathogens identified on culture were Gramnegative bacilli (Escherichia coli (24), Pseudomonas aeruginosa (23), Enterobacter cloacae complex (14), Klebsiella pneumoniae (12), Haemophilus influenzae (11)) and Gram-positive cocci (Staphylococcus aureus (28), Streptococcus pneumoniae (8)) (Table 2). Results of the microbiological analyses according to sample type are described in the supplementary material (Supplementary Table 1). Multiple microorganisms were detected in 33\% of episodes using culture and $43 \%$ using the syndromic rm-PCR method. No influenza virus was found, neither using standard methods nor the syndromic rm-PCR. The syndromic rm-PCR identified 16 antibiotic resistance genes: 11 bla $_{\mathrm{CTX}-\mathrm{M}}$ and $5 \mathrm{mec} A / C$ - MREJ genes. All pathogens identified as harboring an extended-spectrum $\beta$-lactamase on AST $(n=10)$ were found positive for the bla $a_{\text {CTX-M }}$ by the rm-PCR. The 2 Staphylococcus aureus strains resistant to methicillin on AST were detected by the rm-PCR, which detected 2 more methicillin-resistant Staphylococcus aureus strains that were not found on culture. Six Enterobacteriaceae probably had an overproduction of AmpC $\beta$-lactamase, which could not be detected by the rm-PCR.

The empirical therapy included a $\beta$-lactam in 147 (92\%) pneumonia episodes: third-generation cephalosporin $(n=35,22 \%)$, amoxicillin/clavulanate $(n=27,17 \%)$, piperacillin/tazobactam $(n=29,18 \%)$, or fourth-generation cephalosporin $(n=19,12 \%)$, and carbapenems $(n=27,17 \%)$. A companion molecule was prescribed in 79 (50\%) episodes: mainly macrolides $(n=20,13 \%)$, imidazoles $(n=17,11 \%)$, and aminoglycosides $(n=15,9 \%$; Table 3$)$.

Impact of the syndromic rm-PCR on antimicrobial therapy Based on the results of the rm-PCR, the multidisciplinary committee proposed a modification of the routine empirical therapy in 123 (77\%) pneumonia episodes: deescalation in 63 (40\%) and escalation in 35 (22\%;
Table 4). The proportion of antibiotic modifications was higher in VAP $(32 / 37,87 \%)$, as compared to HAP (54/ $68,79 \%)$ and CAP (37/54, 69\%). De-escalation consisted in change of the pivotal drug to a narrower spectrum antibiotic $(n=37,23 \%)$ and/or discontinuation of a companion drug $(n=37,23 \%)$, or discontinuation of all antibiotics $(n=9,6 \%)$. Sputum samples were associated with less antimicrobial modifications $(21 / 33,64 \%)$ compared to other sample types $(58 / 71,82 \% ; 27 / 34,80 \% ; 17 / 21$, $81 \%$; for ETA, BAL, and BBS respectively). Modifications in the sputum group were escalation and de-escalation in the same number of cases, whereas there was more de-escalation compared to escalation in the other type of samples (Supplementary Table 2).

In microbiologically documented episodes $(n=95)$, pathogens were susceptible to the empirical therapy in 73 (77\%) episodes and to the PCR-guided therapy in 83 (87\%) episodes. The proportion of the empirical and PCR-guided therapies that were active on the documented pathogens did not statistically differ between CAP, HAP, and VAP (data not shown). When the PCRguided therapy differed from the empirical therapy, pathogens were susceptible to PCR-guided therapy in $85 \%$ [28/33] of the de-escalations and $88 \%$ [22/25] of the escalations. For 14 (9\%) patients, the PCR-guided therapy was active against documented pathogens, whereas the empirical routine therapy was non-active. Conversely, 4 patients had an active empirical therapy, and the multidisciplinary committee proposed a non-active PCR-guided therapy. In 1 patient, the syndromic rmPCR was negative and the committee proposed to discontinue antibiotics, whereas standard culture grew up $10^{7} \mathrm{CFU} / \mathrm{ml}$ Morganella morganii (not included in the panel). Of note, Gram-negative bacilli were identified on direct examination of the respiratory sample (BAL), but this information was not considered by the multidisciplinary committee. In 2 other patients treated with fourth-generation cephalosporin as empirical therapy, the committee proposed a treatment with piperacillintazobactam. These 2 patients had a syndromic rm-PCR positive for Enterobacter cloacae complex confirmed by culture, consequently found resistant to piperacillintazobactam due to overexpression of the naturally produced AmpC $\beta$-lactamase. The fourth patient was empirically treated with ceftolozane-tazobactam, and the committee proposed to treat him with ceftazidime. Both syndromic rm-PCR and conventional culture identified Pseudomonas aeruginosa, which was phenotypically confirmed as resistant to ceftazidime. Of note, this patient was already known to be colonized with a $P$. aeruginosa of this particular phenotype. Details on all inadequate antibiotic therapies (either empirical or PCR-guided) are available in the supplementary material (Supplementary Table 3). 
Table 1 Characteristics of study participants $(n=159)$

\begin{tabular}{|c|c|c|c|c|}
\hline & Overall $n=159$ & CAP $n=54$ & $\operatorname{HAP} n=68$ & $\operatorname{VAP} n=37$ \\
\hline \multicolumn{5}{|l|}{ Clinical features } \\
\hline \multicolumn{5}{|l|}{ Admission ward } \\
\hline Intensive care unit & $129(81)$ & $41(76)$ & $51(75)$ & $37(100)$ \\
\hline Surgical unit & $13(8)$ & 1 & $12(18)$ & - \\
\hline Medical unit & $12(7)$ & $7(13)$ & $5(7)$ & - \\
\hline Emergency room & $5(4)$ & $5(9)$ & - & - \\
\hline \multicolumn{5}{|l|}{ Demographics } \\
\hline Age, years & $63[55-72]$ & $64[55-74]$ & $64[56-71]$ & $61[53-69]$ \\
\hline Male & $107(67)$ & $32(59)$ & $46(67)$ & $29(78)$ \\
\hline \multicolumn{5}{|l|}{ Comorbid conditions } \\
\hline Immune suppression ${ }^{a}$ & $52(33)$ & $11(20)$ & $29(43)$ & $12(32)$ \\
\hline Chronic pulmonary conditions ${ }^{\mathrm{b}}$ & $52(33)$ & $19(35)$ & $26(38)$ & $7(19)$ \\
\hline Charlson score & $2[1-5]$ & $2[1-4]$ & $3[1-6]$ & $2[1-5]$ \\
\hline \multicolumn{5}{|l|}{ Severity of disease at inclusion } \\
\hline SOFA score (in ICU patients) & $6[4-9]$ & $6[4-9]$ & $6[4-9]$ & $6[4-8]$ \\
\hline Septic shock & $49(31)$ & $13(24)$ & $18(27)$ & $18(49)$ \\
\hline Initiation of respiratory support & $64(40)$ & $20(37)$ & $34(50)$ & - \\
\hline In-hospital death & $45(28)$ & $14(26)$ & $13(19)$ & $18(49)$ \\
\hline \multicolumn{5}{|l|}{ Type of microbiological sampling } \\
\hline Endotracheal aspiration & $71(45)$ & $30(55)$ & $33(49)$ & $8(22)$ \\
\hline Sputum & $33(21)$ & $13(24)$ & $20(29)$ & - \\
\hline Bronchoalveolar lavage & $34(21)$ & $4(7)$ & $13(19)$ & $17(46)$ \\
\hline Blind bronchial sampling & $21(13)$ & $7(13)$ & $2(3)$ & $12(32)$ \\
\hline \multicolumn{5}{|l|}{ Microbiology-conventional techniques } \\
\hline Escherichia coli & $17(11)$ & $4(7)$ & $5(7)$ & $8(22)$ \\
\hline Pseudomonas aeruginosa & $15(9)$ & $3(6)$ & $8(12)$ & $4(11)$ \\
\hline Staphylococcus aureus & $15(9)$ & $3(6)$ & $4(6)$ & $8(22)$ \\
\hline Klebsiella pneumoniae group & $10(6)$ & $2(4)$ & $5(7)$ & $3(8)$ \\
\hline Haemophilus influenzae & $10(6)$ & $4(7)$ & $4(6)$ & $2(5)$ \\
\hline Streptococcus pneumoniae & $6(4)$ & $3(6)$ & $3(4)$ & - \\
\hline Enterobacter cloacae complex & $7(4)$ & 1 & $5(7)$ & $1(3)$ \\
\hline Enterobacter aerogenes & $4(3)$ & 1 & $2(3)$ & 1 \\
\hline Citrobacter freundii & $2(1)$ & - & $2(3)$ & - \\
\hline Serratia marcescens & $2(1)$ & - & 1 & 1 \\
\hline Morganella morganii & $2(1)$ & - & - & $2(5)$ \\
\hline Raoultella & $2(1)$ & 1 & - & 1 \\
\hline Stenotrophomonas maltophilia & $2(1)$ & - & - & $2(5)$ \\
\hline Acinetobacter calcoaceticus-baumannii complex & 1 & - & - & 1 \\
\hline Legionella pneumophila & 1 & 1 & - & - \\
\hline
\end{tabular}

Results are presented as $n$ (\%) or median [interquartile range]

Conventional techniques include culture for all pathogens presented, apart from Legionella pneumophila which was detected using molecular techniques. Only pathogens cultured at pre-defined levels are presented $\left(\geq 10^{3} \mathrm{CFU} / \mathrm{ml}\right.$ for blind bronchial sampling, $\geq 10^{4} \mathrm{CFU} / \mathrm{ml}$ for bronchoalveolar lavage, $\geq 10^{5} \mathrm{CFU} / \mathrm{ml}$ for endotracheal aspiration, and $\geq 10^{7} \mathrm{CFU} / \mathrm{ml}$ for sputum samples)

${ }^{a}$ Immunosuppression was defined as leucopenia $<1$ Giga/L, HIV infection, history of solid organ or stem cell transplantation, and immunosuppressive treatment including corticosteroids over $10 \mathrm{mg} /$ day of equivalent prednisone for more than 15 days

${ }^{\mathrm{b}}$ Chronic pulmonary condition included patients with history of chronic obstructive pulmonary disease, severe bronchiectasis, cystic fibrosis, and chronic respiratory insufficiency 
Table 2 Comparison of microbiological documentation obtained by conventional techniques versus rm-PCR in 159 pneumonia episodes

\begin{tabular}{ll}
\hline Pathogen identification & $\begin{array}{l}\text { Conventional } \\
\text { techniques }\end{array}$ \\
\hline
\end{tabular}

Targets included in the rm-PCR panel

\begin{tabular}{|c|c|c|}
\hline \multicolumn{3}{|l|}{ Gram-negative bacilli } \\
\hline Escherichia coli & 24 & 33 \\
\hline Pseudomonas aeruginosa & 23 & 28 \\
\hline Enterobacter cloacae complex & 13 & 22 \\
\hline Klebsiella pneumoniae group & 13 & 14 \\
\hline Haemophilus influenzae & 11 & 33 \\
\hline $\begin{array}{l}\text { Acinetobacter calcoaceticus-baumannii } \\
\text { complex }\end{array}$ & 3 & 4 \\
\hline Enterobacter aerogenes & 5 & 7 \\
\hline Serratia marcescens & 3 & 5 \\
\hline Proteus spp. & 2 & 8 \\
\hline Moraxella catarrhalis & 1 & 5 \\
\hline Klebsiella oxytoca & 1 & 5 \\
\hline \multicolumn{3}{|l|}{ Gram-positive cocci } \\
\hline Staphylococcus aureus & 26 & 42 \\
\hline Streptococcus pneumoniae & 8 & 16 \\
\hline Streptococcus pyogenes & 0 & 0 \\
\hline Streptococcus agalactiae & 0 & 0 \\
\hline \multicolumn{3}{|l|}{ Atypical bacteria } \\
\hline Legionella pneumophila & 1 & 1 \\
\hline Mycoplasma pneumoniae & 0 & 1 \\
\hline Chlamydophila pneumoniae & 0 & 0 \\
\hline \multicolumn{3}{|l|}{ Resistance } \\
\hline mecA & & 5 \\
\hline CTX-M & & 11 \\
\hline \multicolumn{3}{|l|}{ argets not included in the rm-PCR panel } \\
\hline Stenotrophomonas maltophilia & 4 & - \\
\hline Morganella morganii & 3 & - \\
\hline Raoultella ornithinolytica & 3 & - \\
\hline Citrobacter sp. & 4 & - \\
\hline Hafnia & 1 & \\
\hline Others* & 11 & - \\
\hline
\end{tabular}

Conventional techniques include culture for all pathogens presented, apart from Legionella pneumophila which was detected using molecular techniques $r m-P C R$ real-time multiplex polymerase chain reaction

*Acinetobacter junii $(n=1)$, Actinomyces odontolyticus $(n=1)$, Corynebacterium sp. $(n=1)$, coagulase-negative Staphylococcus $(n=4)$, Enterococcus faecium $(n=1)$, Enterococcus faecalis $(n=6)$, Lactobacillus reuteri $(n=1)$, Streptococcus alpha $(n=1)$, Streptococcus pseudopneumoniae $(n=1)$

Overall, as described in Table 3, compared to routine empirical therapy, the syndromic rm-PCR would have theoretically led to avoid 27 prescriptions of $\beta$-lactams (third-generation cephalosporins $[n=15]$, carbapenems $[n=8]$, amoxicillin-clavulanate $[n=2]), 29$ prescriptions
Table 3 Comparison of antimicrobial therapies prescribed in the empirical and the rm-PCR-guided groups $(n=159)$

\begin{tabular}{|c|c|c|}
\hline Antibiotic treatment & $\begin{array}{l}\text { Routine empirical } \\
\text { therapy (\%) }\end{array}$ & $\begin{array}{l}\text { rm-PCR-guided } \\
\text { therapy (\%) }\end{array}$ \\
\hline Beta-lactams & $147(92)$ & $131(82)$ \\
\hline Third-generation cephalosporin & $35(22)$ & $24(15)$ \\
\hline Piperacillin-tazobactam & $29(18)$ & $34(21)$ \\
\hline Amoxicillin-clavulanate & $27(17)$ & $25(16)$ \\
\hline Carbapenem & $27(17)$ & $19(12)$ \\
\hline Cefepime & $19(12)$ & $19(12)$ \\
\hline Amoxicillin & $5(3)$ & $4(3)$ \\
\hline Ceftazidime & $3(2)$ & $7(4)$ \\
\hline Cefazolin & $2(1)$ & $6(4)$ \\
\hline Penicillin M & $0(0)$ & $2(1)$ \\
\hline Companion molecule & $79(50)$ & $50(31)$ \\
\hline Macrolide & $20(13)$ & $4(3)$ \\
\hline Metronidazole & $17(11)$ & $8(5)$ \\
\hline Aminoglycoside & $15(9)$ & $19(12)$ \\
\hline Fluoroquinolone & $8(5)$ & $5(3)$ \\
\hline Glycopeptide & $6(4)$ & $2(1)$ \\
\hline Linezolide & $6(4)$ & $8(5)$ \\
\hline Cotrimoxazole & $7(4)$ & $4(3)$ \\
\hline Antifungal therapy & $5(3)$ & $4(3)$ \\
\hline Oseltamivir & $3(2)$ & 0 \\
\hline Other & $4(3)$ & 0 \\
\hline
\end{tabular}

Results are presented as $n$ (\%)

of companion antibiotics (macrolides, metronidazole, fluoroquinolones, glycopeptides, cotrimoxazole), and 8 other molecules (antifungal therapy, oseltamivir, other beta-lactams). It would however have increased the number of prescriptions of piperacillin-tazobactam $(n=$ $5)$, ceftazidime $(n=4)$, cefazolin $(n=4)$, and penicillin $M$ $(n=2)$.

\section{Discussion}

In this retrospective multicenter study, a syndromic rmPCR approach with semi-quantitative results had the potential to lead to a change in empirical antimicrobial therapy in $77 \%$ of pneumonia episodes in adult patients. The most frequent intervention was a de-escalation, which occurred in almost half of the patients. Using standard culture and AST as the reference method, PCR-guided antibiotic treatment was more frequently adequate when compared to the empirical treatment.

As observed in previous studies, microbiological documentation was almost twice as high using the rm-PCR compared to the standard method [14]. If this higher sensitivity is an advantage for patients treated with antibiotics prior to sampling, it also implies a very cautious interpretation of results. The test might detect nucleic acids from 
Table 4 Impact of the rm-PCR results on antibiotic prescription, according to the multidisciplinary committee $(n=159)$

\begin{tabular}{lllll}
\hline & Overall, $n=159$ & CAP, $n=54$ & HAP, $n=68$ & VAP, $n=37$ \\
\hline Antibiotic modification & $123(77)$ & $37(69)$ & $54(79)$ & $32(87)$ \\
De-escalation & $63(40)$ & $20(37)$ & $25(37)$ & $18(49)$ \\
Escalation & $35(22)$ & $8(15)$ & $18(27)$ & $9(24)$ \\
Undetermined & $25(16)$ & $9(17)$ & $11(16)$ & $5(14)$ \\
No change & $36(23)$ & $17(32)$ & $14(21)$ & $5(14)$ \\
\hline
\end{tabular}

Results are presented as $n(\%)$

$C A P$ community-acquired pneumonia, HAP hospital-acquired pneumonia, VAP ventilator-associated pneumonia

dead pathogens that are not involved in the actual pneumonia episode. As there is no scientific data regarding the clearance of bacterial genomic material from the lung, this could lead to overtreatment of non-viable microorganisms. Similarly, distinguishing colonization from infection is challenging and requires interpreting the results in accordance with the clinical context. Even if semi-quantitative quantification of bacterial targets provided by the BioFire ${ }^{\circ}$ FilmArray ${ }^{\circ}$ Pneumonia Panel is helpful to guide the decision, there are currently no consensual thresholds of clinical significance for molecular methods. In the present report, we did not evaluate the correlation between culture quantification (in CFU/ml) and molecular semi-quantification (in DNA copies $/ \mathrm{ml}$ ). This question is currently under investigation [15]. Meanwhile, clinicians should be aware that molecular diagnosis is highly sensitive and the use of thresholds established for conventional culture to guide interpretation of the rm-PCR is not straightforward and may lead to misinterpretation.

It should also be kept in mind that even if it detects a large panel of microorganisms, the test is not exhaustive and several important pathogens (such as Morganella spp., Citrobacter spp., Hafnia alvei, Stenotrophomonas maltophilia, or Pneumocystis jirovecii) are not included. The same issue can be raised for the resistance genes, especially ESBL-encoding genes for which only the most frequent ones (i.e., bla $a_{\mathrm{CTX}-\mathrm{M}}$ ) are included in the panel. Thus, when a pathogen is identified by the test, the absence of resistance genes does not predict with certainty its phenotypic susceptibility. When a gene encoding for a resistance marker is detected alongside with different bacteria, it is not possible to link the resistance to one of the detected microorganisms. Also, overexpression of intrinsic resistance genes (such as AmpC $\beta$-lactamase) is not detected by the panel. From this perspective, rmPCR should not be considered as a sufficient diagnostic test for pneumonia but rather as a complementary tool in adjunction to standard culture and AST, to allow for an earlier pathogen-directed therapy. Multidisciplinary discussion of the results is a prerequisite for optimal use. In our experience, the rare errors (4 over 159 episodes) in the choice of PCR-guided therapy were linked to (1) Amp-C overproduction in Enterobacter sp. in 2 cases,
(2) isolation of pathogens not included in the panel (i.e., Morganella morganii) in 1 case, and (3) isolation of a multi-drug-resistant $P$. aeruginosa (that was previously isolated in this particular patient) in 1 case. In this last case, it is important to note that interpretation of the rm-PCR in light of the previous microbial documentation could have prevented this error.

Our intervention was based on a multidisciplinary approach with physicians from different specialties interpreting the results of the rm-PCR together and agreeing on an optimal therapy. The success of this approach is concordant with previous studies where strategies combining rapid diagnostic testing and AMS interventions in bloodstream infections showed a significant and synergistic impact on early antibiotic de-escalation, mortality, and costs [23-26]. Moreover, implementation of AMS programs, led by dedicated multidisciplinary teams, is strongly encouraged to improve the quality of antibiotic prescriptions [27-29]. Interestingly, the use of rapid diagnostic tests without active AMS intervention failed to provide the expected benefits in patients [26, 30-32]. It is uncertain whether the same results would have been observed if only one clinician, non-expert of the diagnostic methods, had to choose alone a therapy guided by the rm-PCR results. Another strength of the present study is its multicenter character, as it improves the generalizability of the results, but also because the multidisciplinary committees were local and therefore aware of the ecology of their hospitals.

The present study presents some limitations. Its retrospective and observational design did not allow comparison of clinical outcomes with the rm-PCR versus standard treatment approach. Second, although we included a large panel of pneumonia patients (severe ICU patients with VAP or HAP and non-severe CAP patients from the emergency room), it remains unclear what group of patients will benefit most from the syndromic rm-PCR diagnosis. Further studies should evaluate this new test in more selected groups of patients. However, most patients herein were severe patients hospitalized in ICUs with complicated microbiological histories, and results suggest the rm-PCR could help to choose the antimicrobial treatment even in this particular group of 
patients. Also, even if we tried to give the multidisciplinary committee as much information as needed to choose the antibiotic treatment, in some cases, important data may have been missing. As an example, this might explain why the clinician chose a ceftolozane-tazobactambased treatment for a patient with a past history of infection with piperacillin-tazobactam-resistant $P$. aeruginosa, whereas the committee decided to use piperacillintazobactam despite identification of $P$. aeruginosa by the rm-PCR. Again, this reinforces the necessity to carefully interpret the rm-PCR report, in light of the clinical and historical microbiological data. In our panel of CAP patients, only half had microbiological documentation on conventional culture. This is in accordance with previous studies that highlighted a low rate of documentation in CAP, due to difficulties to obtain contributive microbiological samples and to previous exposure to antibiotics [6]. In the 27 CAP patients with positive microbiology results, we identified 10 pathogens frequently involved in CAP (Staphylococcus aureus, Haemophilus influenzae, Streptococcus pneumoniae) and 12 Gram-negative bacilli (4 Escherichia coli, 3 Pseudomonas aeruginosa, 2 Klebsiella pneumoniae, 2 Enterobacter sp., 1 Raoultella). The number of Gram-negative bacteria isolated is likely related to the profile of patients included in our study. Indeed, all participating centers were tertiary university hospitals, referral centers for specific comorbidities as highlighted in Table 1: among CAP patients, $35 \%$ had chronic respiratory conditions and $20 \%$ immune suppression.

The gold standard used herein for microbiological diagnosis was culture with AST. In certain cases, the syndromic rm-PCR detected pathogens that were not detected by culture. This finding questions the choice of culture as the gold standard for microbial identification, notably in case of antibiotic treatment before sampling. Because the molecular diagnostic methods are probably more sensitive than cultures, the present results would have been even more favorable for PCR-guided therapy if we had also considered as documentation the organisms identified by rm-PCR and not by culture. As an example, one patient had a Staphylococcus aureus (10 $0^{5}$ copies $\left./ \mathrm{mL}\right)$, a Moraxella catarrhalis $\left(10^{4}\right.$ copies/ $\mathrm{mL}$ ), and a mecA/C-MREJ gene found by rm-PCR while the culture remained negative and was therefore considered as "undocumented." Also, because two thirds of our samples were non-protected (ETA and sputum), this could have led to an over-identification of bacteria [33]. However, since we used the culture and validated the significance thresholds for interpretation, this bias was minimized. The high proportion of non-invasive samples reflects the heterogeneity of practices between ICUs and is consistent with the IDSA guidelines which suggest noninvasive sampling with semi-quantitative cultures to diagnose VAP, rather than invasive sampling [4].

Many questions regarding the syndromic rm-PCR still remain unanswered and should be addressed in future evaluations. The cost-effectiveness ratio of the syndromic rm-PCR needs to be evaluated in order to justify its use in particular subgroups of patients. To date, no studies addressed this medico-economic outcome in lower respiratory tract infections, but a syndromic rmPCR developed for the higher tract respiratory infections was found to decrease the length of hospital stay, duration of antibiotic therapy, and time in isolation, therefore decreasing related costs [34, 35]. As previously discussed, thresholds for interpretation of rm-PCR results cannot be directly derived from those established for culture and must be more precisely defined. Because of these limits, physicians should be aware of the test's drawbacks and the present results highlight the need for a close cooperation between infectious disease specialists, clinical microbiologists, and clinicians for the interpretation of results. We also suggest that the results of the test should be included in a decision algorithm, alongside with the suspected pathogens, the history of the patient, and their personal risk factors for MDRO infection. We recommend that decision regarding antibiotic treatment based on rm-PCR results should consider clinical presentation, local epidemiology, direct smear of the sample, and historical microbiological data in each patient. As a guidance for use in clinical practice and based on our experience, we may recommend to (1) consider the semi-quantitative result regarding the type of sample, in order to discriminate between colonization and infection (future results should be available soon to precise the significance thresholds); (2) if a microorganism is identified and thought to be pathogenic, the antimicrobial therapy should be chosen considering colonization of the patient, previous microbial documentations, and risk factors for MDRO; (3) if a pathogen of the group 3 is detected, an Amp-C overproduction cannot be excluded and therefore treatment with cefepim or meropenem should be considered; (4) oseltamivir should not be prescribed in the absence of influenza detection; (5) a companion molecule such as macrolide could be discontinued for community-acquired pneumonia without identification of atypical bacteria; and (6) identification of a resistance gene should always be considered.

To date, the rm-PCR should not be considered as a sufficient diagnostic test for pneumonia but rather as a complementary tool in adjunction to standard culture and AST, to allow for an earlier pathogen-directed therapy. Controlled randomized trials evaluating the impact of rm-PCR on patient's outcomes are currently ongoing, and their results will be of major help to precise the 
impact of the rm-PCR on infection control, length of stay, and resistance selection.

\section{Conclusion}

Results suggest that early use of rm-PCR in pneumonia could reduce unnecessary antimicrobial exposure without compromising the appropriateness of the treatment. Together with an expert advice, this promising diagnostic tool could improve the quality of care and participate in the reduction of broad-spectrum antimicrobial agents' use. Prospective randomized controlled trials are needed to confirm these results, identify categories of patients that would most benefit from the test, and define precise guidelines to appropriately adjust the empirical therapy based on the results.

\section{Supplementary information}

Supplementary information accompanies this paper at https://doi.org/10. 1186/s13054-020-03114-y.

\section{Additional file 1: Supplementary table 1. Microbiological} documentation depending on the sample type $(n=159)$. Supplementary table 2. Impact of the rm-PCR results on antibiotic prescription, according to the multidisciplinary committee and regarding sample type ( $n=$ 159). Supplementary Table 3 . Description of inadequate antibiotic regimen.

\section{Abbreviations \\ AMS: Antimicrobial stewardship program; AST: Antimicrobial susceptibility testing; BAL: Bronchoalveolar lavage; CAP: Community-acquired pneumonia; HAP: Hospital-acquired pneumonia; ICU: Intensive care unit; MDRO: Multidrug-resistant organism; rm-PCR: Real-time multiplex PCR; VAP: Ventilator-acquired pneumonia}

\section{Acknowledgements}

The authors thank all research teams and laboratory teams that participated in the study in each center.

The authors thank Philip Robinson/Verena Landel for participating in manuscript preparation.

The authors thank all the members from the ADAPT study group: Agathe Becker, Julien Charpentier, Julien Textoris, Claude-Alexandre Gustave, Grégory Destras, François Vandenesch, Bruno Lina, Jean Sebastien Casalegno, Manon Lejeune, Philippe Montravers, Claire Poyart, Hugo Tête, Jean-François Timsit, and Thomas Uberti.

\section{Authors' contributions}

CM and SK elaborated the study protocol, had full access to all of the data, did the analysis, and take responsibility for the integrity of the data and the accuracy of the data analysis. CM, OD, VC, LAL, and SK drafted the paper. All authors critically revised the manuscript and gave final approval for the version to be published.

\section{Funding}

Not applicable.

\section{Availability of data and materials}

The datasets used during the current study are available from the corresponding author on reasonable request.

\section{Ethics approval and consent to participate}

According to the French legislation, this study was approved by the Ethics Committee of the French Society of Infectious Diseases (Société de Pathologie Infectieuse de Langue Française), n²019-0902, and declared to the French national data protection commission (Commission Nationale de I'Informatique et des Libertés, CNIL), n¹9-213.
Consent for publication

Not applicable.

\section{Competing interests}

bioMerieux SA provided IUO tests for the study. bioMerieux SA did not interfere with data collection and analysis, nor with manuscript preparation. Dr. Julien Textoris participated in the elaboration of the protocol. SK declares grants from bioMérieux and lecture fees and conference invitations from bioMérieux, MSD, and Accelerate Diagnostics. $C M$ received lecture fees from bioMerieux SA.

ATD and NG report no conflicts of interest.

VC reports personal fees from Accelerate Diagnostics, Astellas, bioMérieux,

Correvio, Curetis, Eumédica, Menarini, Mylan, Pfizer, and Sanofi.

$L A$ received lecture fees from Pfizer, MSD, Biocodex, and Biomerieux.

All authors have submitted the ICMJE form for disclosure of potential conflicts of interest. Conflicts that the editors consider relevant to the content of the manuscript have been disclosed.

\section{Author details}

'Département d'Anesthésie et Réanimation, Hospices Civils de Lyon, Hôpital E. Herriot, Lyon, France. ${ }^{2}$ Service de Réanimation Médicale Infectieuse, APHP, Hôpital Bichat Claude Bernard, Paris, France. ${ }^{3}$ Service de Bactériologie-Hygiène Hospitalière, CHU de Rennes, Rennes, France. ${ }^{4} \mathrm{CNR}$ de la Résistance aux Antibiotiques (Laboratoire Associé Entérocoques), Rennes, France. ${ }^{5}$ Equipe Mobile d'Infectiologie, APHP, Hôpital Cochin, Centre Université de Paris, Paris, France. 'Département d'anesthésie-réanimation, APHP, Hôpital Bichat-Claude Bernard, Université de Paris, Paris, France. ${ }^{7}$ Inserm U 1148 LVTS, Université de Paris, Paris, France. ${ }^{8}$ Service de Bactériologie, APHP, Hôpital Cochin, Centre Université de Paris, Paris, France. ${ }^{9}$ Service de Bactériologie, APHP Nord, Université de Paris, Hôpital Bichat, Paris, France. ${ }^{10}$ IAME, INSERM, Université de Paris, Paris, France. ${ }^{11}$ Service de Maladies Infectieuses et Réanimation Médicale, Hôpital Pontchaillou, Université de Rennes, Rennes, France. ${ }^{12}$ Faculté de Médecine, Université de Rennes 1, Unité INSERM CIC 1414, IFR 140, Rennes, France. ${ }^{13}$ Equipe mobile d'infectiologie, Hospices Civils de Lyon, Hôpital E. Herriot, Lyon, France.

${ }^{14}$ Service de Maladies Infectieuses et Réanimation Médicale, CHU Rennes, Rennes, France. ${ }^{15}$ Service de Bactériologie, AP-HP Centre, Hôpital Cochin, Université de Paris, Paris, France. ${ }^{16}$ Plateau de Microbiologie 24/24, Institut des Agents Infectieux, Hospices Civils de Lyon, Centre de Biologie et Pathologie Nord, Lyon, France. ${ }^{17}$ INSERM CIRI LYON, Equipe "Pathogénie des Staphylocoques", Lyon, France. ${ }^{18} \mathrm{CNR}$ de la Résistance aux Antibiotiques (laboratoire associé 'Entérocoques), Rennes, France. ${ }^{19}$ Unité Inserm U1230, Université de Rennes 1, Rennes, France.

Received: 23 April 2020 Accepted: 29 June 2020

Published online: 14 July 2020

\section{References}

1. Liu VX, Fielding-Singh V, Greene JD, Baker JM, Iwashyna TJ, Bhattacharya J, et al. The timing of early antibiotics and hospital mortality in sepsis. Am J Respir Crit Care Med. 2017;196:856-63.

2. Yokota PKO, Marra AR, Martino MDV, Victor ES, Durão MS, Edmond MB, et al. Impact of appropriate antimicrobial therapy for patients with severe Sepsis and septic shock - a quality improvement study. PLoS One. 2014;9:110.

3. Dellinger RP, Levy MM, Rhodes A, Annane D, Gerlach H, Opal SM, et al. Surviving Sepsis Campaign: international guidelines for management of severe sepsis and septic shock, 2012. Intensive Care Med. 2013;39:165-228.

4. Kalil AC, Metersky ML, Klompas M, Muscedere J, Sweeney DA, Palmer LB, et al. Management of adults with hospital-acquired and ventilatorassociated pneumonia: 2016 clinical practice guidelines by the Infectious Diseases Society of America and the American Thoracic Society. Clin Infect Dis. 2016;63:e61-111.

5. Torres A, Niederman MS, Chastre J, Ewig S, Fernandez-Vandellos P, Hanberger $\mathrm{H}$, et al. International ERS/ESICM/ESCMID/ALAT guidelines for the management of hospital-acquired pneumonia and ventilator-associated pneumonia: guidelines for the management of hospital-acquired pneumonia (HAP)/ventilator-associated pneumonia (VAP) of the European Respiratory Society (ERS), European Society of Intensive Care Medicine (ESICM), European Society of Clinical Microbiology and Infectious Diseases 
(ESCMID) and Asociación Latinoamericana del Tórax (ALAT). Eur Respir J. 2017:50:1700582.

6. Jain S, Self WH, Wunderink RG, CDC EPIC study team. Community-acquired pneumonia requiring hospitalization. N Engl J Med. 2015;373:2382.

7. Messika J, Stoclin A, Bouvard E, Fulgencio J-P, Ridel C, Muresan I-P, et al. The challenging diagnosis of non-community-acquired pneumonia in nonmechanically ventilated subjects: value of microbiological investigation. Respir Care. 2016;61:225-34.

8. Versporten A, Zarb P, Caniaux I, Gros M-F, Drapier N, Miller M, et al. Antimicrobial consumption and resistance in adult hospital inpatients in 53 countries: results of an internet-based global point prevalence survey. Lancet Glob Health. 2018;6:e619-29.

9. Ramanan P, Bryson AL, Binnicker MJ, Pritt BS, Patel R. Syndromic panelbased testing in clinical microbiology. Clin Microbiol Rev. 2017;31:1-28.

10. Bauer KA, Perez KK, Forrest GN, Goff DA. Review of rapid diagnostic tests used by antimicrobial stewardship programs. Clin Infect Dis. 2014;59(Suppl 3):S134-45.

11. Rappo U, Schuetz AN, Jenkins SG, Calfee DP, Walsh TJ, Wells MT, et al. Impact of early detection of respiratory viruses by multiplex PCR assay on clinical outcomes in adult patients. J Clin Microbiol. 2016;54:2096-103.

12. Gadsby NJ, Russell CD, McHugh MP, Mark H, Conway Morris A, Laurenson IF, et al. Comprehensive molecular testing for respiratory pathogens in community-acquired pneumonia. Clin Infect Dis. 2016;62:817-23.

13. Yugueros-Marcos J, Barraud O, lannello A, Ploy MC, Ginocchio C, Rogatcheva $\mathrm{M}$, et al. New molecular semi-quantification tool provides reliable microbiological evidence for pulmonary infection. Intensive Care Med. 2018:44:2302-4.

14. Lee SH, Ruan S-Y, Pan S-C, Lee T-F, Chien J-Y, Hsueh P-R. Performance of a multiplex PCR pneumonia panel for the identification of respiratory pathogens and the main determinants of resistance from the lower respiratory tract specimens of adult patients in intensive care units. J Microbiol Immunol Infect. 2019:52:920-8.

15. Gastli N, Alviset S, Billoët A, et al. Evaluation of the FILMARRAY Pneumonia Panel for rapid microbiological diagnosis of pneumonia [abstract P1560]. In: Program and abstracts of the 29th European Congress of Clinical Microbiology and Infectious Diseases (Amsterdam). 2019.

16. Guidelines for the management of adults with hospital-acquired, ventilatorassociated, and healthcare-associated pneumonia. Am J Respir Crit Care Med. American Thoracic Society - AJRCCM; 2005;171:388-416.

17. Metlay JP, Waterer GW, Long AC, Anzueto A, Brozek J, Crothers K, et al. Diagnosis and treatment of adults with community-acquired pneumonia. An official clinical practice guideline of the American Thoracic Society and Infectious Diseases Society of America. Am J Respir Crit Care Med. 2019;200: e45-67.

18. Recommendations 2019 by the European Committee on antimicrobial susceptibility reading EUCAST Available at: https://www.sfm-microbiologie. org/wp-content/uploads/2019/02/CASFM2019_V1.0.pdf Accessed 23 Jan 2020.

19. Rouby JJ, Martin De Lassale E, Poete P, Nicolas MH, Bodin L, Jarlier V, et al. Nosocomial bronchopneumonia in the critically ill. Histologic and bacteriologic aspects. Am Rev Respir Dis. 1992;146:1059-66.

20. De Bus L, Denys W, Catteeuw J, Gadeyne B, Vermeulen K, Boelens J, et al. Impact of de-escalation of beta-lactam antibiotics on the emergence of antibiotic resistance in ICU patients: a retrospective observational study. Intensive Care Med. 2016;42:1029-39.

21. Paul M, Dickstein Y, Raz-Pasteur A. Antibiotic de-escalation for bloodstream infections and pneumonia: systematic review and meta-analysis. Clin Microbiol Infect. 2016;22:960-7.

22. Weiss E, Zahar J-R, Lesprit P, Ruppe E, Leone $\mathrm{M}$, Chastre J, et al. Elaboration of a consensual definition of de-escalation allowing a ranking of $\beta$-lactams. Clin Microbiol Infect. 2015;21:649 e1-10.

23. Banerjee R, Teng CB, Cunningham SA, Ihde SM, Steckelberg JM, Moriarty JP, et al. Randomized trial of rapid multiplex polymerase chain reaction-based blood culture identification and susceptibility testing. Clin Infect Dis. 2015; 61:1071-80.

24. Bookstaver PB, Nimmich EB, Smith TJ, Justo JA, Kohn J, Hammer KL, et al. Cumulative effect of an antimicrobial stewardship and rapid diagnostic testing bundle on early streamlining of antimicrobial therapy in gramnegative bloodstream infections. Antimicrob Agents Chemother. 2017;61: e00189-17.
25. Perez KK, Olsen RJ, Musick WL, Cernoch PL, Davis JR, Peterson LE, et al. Integrating rapid diagnostics and antimicrobial stewardship improves outcomes in patients with antibiotic-resistant Gram-negative bacteremia. J Inf Secur. 2014;69:216-25.

26. Vardakas KZ, Anifantaki Fl, Trigkidis KK, Falagas ME. Rapid molecular diagnostic tests in patients with bacteremia: evaluation of their impact on decision making and clinical outcomes. Eur J Clin Microbiol Infect Dis. 2015; 34:2149-60.

27. Barlam TF, Cosgrove SE, Abbo LM, MacDougall C, Schuetz AN, Septimus EJ, et al. Implementing an Antibiotic Stewardship Program: guidelines by the Infectious Diseases Society of America and the Society for Healthcare Epidemiology of America. Clin Infect Dis. 2016;62:e51-77.

28. Pulcini C, Morel CM, Tacconelli E, Beovic B, de With $\mathrm{K}$, Goossens $\mathrm{H}$, et al. Human resources estimates and funding for antibiotic stewardship teams are urgently needed. Clin Microbiol Infect. 2017:23:785-7.

29. De Waele JJ, Akova M, Antonelli M, Canton R, Carlet J, De Backer D, et al. Antimicrobial resistance and antibiotic stewardship programs in the ICU: insistence and persistence in the fight against resistance. A position statement from ESICM/ESCMID/WAAAR round table on multi-drug resistance. Intensive Care Med. 2018:44:189-96.

30. Terp S, Krishnadasan A, Bowen W, Joo J, Furoy D, Chan J, et al. Introduction of rapid methicillin-resistant Staphylococcus aureus polymerase chain reaction testing and antibiotic selection among hospitalized patients with purulent skin infections. Clin Infect Dis. 2014;58:e129-32.

31. Cosgrove SE, Li DX, Tamma PD, Avdic E, Hadhazy E, Wakefield T, et al. Use of PNA FISH for blood cultures growing Gram-positive cocci in chains without a concomitant antibiotic stewardship intervention does not improve time to appropriate antibiotic therapy. Diagn Microbiol Infect Dis. 2016;86:86-92.

32. Holtzman C, Whitney D, Barlam T, Miller NS. Assessment of impact of peptide nucleic acid fluorescence in situ hybridization for rapid identification of coagulase-negative staphylococci in the absence of antimicrobial stewardship intervention. J Clin Microbiol. 2011:49:1581-2.

33. Sole Violan J, Fernandez JA, Benitez AB, et al. Impact of quantitative invasive diagnostic techniques in the management and outcome of mechanically ventilated patients with suspected pneumonia. Crit Care Med. 2000;28: 2737-41.

34. Rogers BB, Shankar P, Jerris RC, Kotzbauer D, Anderson EJ, Watson JR, et al. Impact of a rapid respiratory panel test on patient outcomes. Arch Pathol Lab Med. 2015;139:636-41.

35. Brendish NJ, Malachira AK, Armstrong L, Houghton R, Aitken S, Nyimbili E, et al. Routine molecular point-of-care testing for respiratory viruses in adults presenting to hospital with acute respiratory illness (ResPOC): a pragmatic, open-label, randomised controlled trial. Lancet Respir Med. 2017:5:401-11.

\section{Publisher's Note}

Springer Nature remains neutral with regard to jurisdictional claims in published maps and institutional affiliations.

\section{Ready to submit your research? Choose BMC and benefit from:}

- fast, convenient online submission

- thorough peer review by experienced researchers in your field

- rapid publication on acceptance

- support for research data, including large and complex data types

- gold Open Access which fosters wider collaboration and increased citations

- maximum visibility for your research: over $100 \mathrm{M}$ website views per year

At BMC, research is always in progress.

Learn more biomedcentral.com/submission 